\title{
Resultados del Tratamiento Quirúrgico de la Perforación Intestinal Causada por Ingestión de Mondadientes. Aportación de 3 Casos
}

\author{
Results of Surgical Treatment of Intestinal Perforation \\ Caused by Toothpick Ingestion. Contribution of 3 Cases
}

Carlos Manterola ${ }^{1,2}$

MANTEROLA, C. Resultados del tratamiento quirúrgico de la perforación intestinal causada por ingestión de mondadientes. Aportación de 3 casos. Int. J. Morphol., 38(5):1212-1216, 2020.

RESUMEN: La ingestión de mondadientes (IM) constituye un evento poco frecuente (se han publicado alrededor de 157 casos a nivel mundial), pero puede causar perforaciones intestinales (PI), con peritonitis, sepsis e incluso muerte. Este hecho, determina la necesidad de intervenir quirúrgicamente a la mayoría de estos pacientes. El objetivo de este manuscrito fue informar resultados del tratamiento quirúrgico de 3 casos de PI por IM. Caso 1: hombre de 52 años, con perforación duodenal y de la vesícula biliar. Caso 2: Mujer de 54 años con perforación cecal. Caso 3: hombre de 72 años, con perforación de colon izquierdo y lesión esplénica. Todos fueron hospitalizados por dolor abdominal y fiebre. En dos casos el diagnóstico se verificó por tomografía y en uno por ecotomografía. Los tres casos fueron intervenidos quirúrgicamente a través de laparotomía. Intervenciones: Caso 1: se realizó colecistectomía y sutura duodenal. Caso 2: se realizó hemicolectomía derecha e ileotransverso anastomosis. Caso 3: se realizó hemicolectomía izquierda y esplenectomía. En todos los casos se encontró el mondadientes. Todos los pacientes evolucionaron de forma satisfactoria, sin complicaciones postoperatorias. La IPD es una emergencia quirúrgica. Las PI son comunes y la peritonitis asociada es frecuente. El pronóstico depende de un diagnóstico precoz y un tratamiento oportuno.

PALABRAS CLAVE: Perforación intestinal; Cirugía; Palillos de dientes; Mondadientes.

\section{INTRODUCCIÓN}

La ingestión de cuerpos extraños (CE) ocurre con cierta frecuencia en la vida cotidiana, a menudo al comer, a la hora de comer o cuando las personas mayores y los niños se llevan objetos extraños a la boca; constituyendo causa de consulta relativamente frecuente en los servicios de urgencia (Su et al., 2014).

En la mayoría de los casos, los CE ingeridos pasarán a través del tubo digestivo de forma espontánea, sin generar problemas (Nicolodi et al., 2016). No obstante ello, en algunas ocasiones, los CE ingeridos pueden impactarse en la mucosa de algún segmento de intestino provocando una reacción inflamatoria y eventualmente una perforación intestinal (PI) (Lunsford \& Sudan, 2012). Los CE que con mayor frecuencia causan PI son huesos de pescado, de pollo y mondadientes (Steinbach et al., 2014).
La mayoría de los casos de ingestión de mondadientes (IM), ocurre durante las comidas; y los factores de riesgo descritos son el hábito de masticar mondadientes, comer alimentos que contienen mondadientes y beber bebidas alcohólicas a la hora de las comidas (Lim et al., 2019). Al respecto, existe evidencia que sustenta el hecho que la IM puede ocasionar una PI en el $80 \%$ de los casos; y que llegan al colon, sólo en $37 \%$ de los pacientes (Steinbach et al.); por otra parte, Hashash et al. (2015) sostienen que la PI solamente se produciría en alrededor del $1 \%$ de los casos. De todos modos, lo que parece lógico es que la PI ocurrirá con mayor probabilidad tras la ingestión de $\mathrm{CE}$ afilados y alargados, como es el caso de los moldadientes; los que se impactan con más frecuencia en puntos de angulación fisiológica o estrechamiento del intestino delgado (Su et al.).

\footnotetext{
${ }^{1}$ Programa de Doctorado en Ciencias Médicas, Universidad de La Frontera, Temuco, Chile.

${ }^{2}$ Centro de Estudios Morfológicos y Quirúrgicos (CEMyQ), Universidad de La Frontera, Temuco, Chile.
} 
Establecer el diagnóstico precoz de una PI causada por la IM es difícil debido a la baja tasa de sospecha (se han publicado alrededor de 157 casos a nivel mundial), porque los pacientes suelen omitir el dato de la IM, y la precisión de las pruebas diagnósticas es baja; todo lo cual retrasa el diagnóstico y la oportunidad del tratamiento.

El objetivo de este manuscrito fue informar resultados del tratamiento quirúrgico de 3 casos de PI por IM.

\section{PRESENTACIÓN DE LOS CASOS}

Caso 1: Sujeto masculino de 52 años, hospitalizado en urgencias por un cuadro de dos días de evolución caracterizado por dolor abdominal intenso y sensación febril. Antecedentes de hipertensión arterial. Al ingreso, se verificó un paciente taquicárdico (108 x min.), febril ( $\mathrm{t}^{\circ}$ axilar de $38,5^{\circ} \mathrm{C}$ ), hipotenso $(100 / 55 \mathrm{mmHg})$, con abdomen sensible en cuadrante superior derecho. Las pruebas de laboratorio del ingreso revelaron leucocitosis de $18200 \times \mathrm{mm}^{3}$, hemoglobina de $12,5 \mathrm{~g} / \mathrm{dL}$ y PCR de 44,7 mg/L. La tomografía axial computarizada (TAC) de abdomen de urgencia, permitió verificar una colección un CE protruyendo por la segunda porción duodenal, en cercanía a la vesícula biliar (Fig. 1). Una vez con cobertura antibiótica, el paciente fue intervenido quirúrgicamente, verificándose una colección de bilis periduodenal, por perforación de la vesícula biliar y del duodeno, por un mondadientes. Luego de un aseo de la cavidad con solución salina, se procedió a una colecistectomía y sutura de la perforación duodenal. El paciente se recuperó de forma adecuada, fue dado de alta al $4^{\circ}$

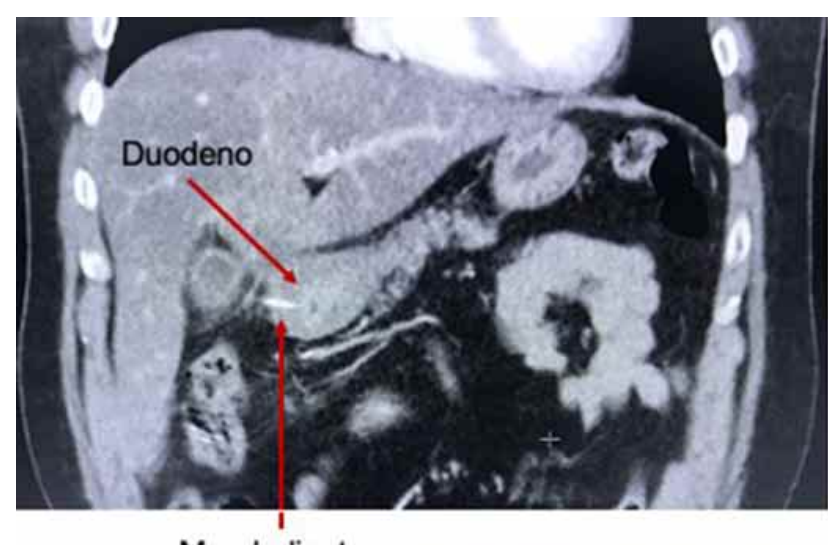

Mondadientes día postoperatorio. Interrogado de forma dirigida en el postoperatorio, el paciente recordó haber ingerido carne envuelta en mondadientes.

Caso 2: Paciente de sexo femenino, de 54 años de edad hospitalizada en urgencias por un cuadro de 4 días de evolución caracterizado por dolor abdominal, astenia, anorexia, deshidratación y fiebre. No tenía antecedentes médicos ni quirúrgicos. A la exploración del ingreso, se verificó una paciente taquicárdica ( $99 \times \mathrm{min})$, febril ( $\mathrm{t}^{\mathrm{o}}$ axilar de $\left.38,6^{\circ} \mathrm{C}\right)$, hipotensa $(98 / 63 \mathrm{mmHg})$, deshidratada, con un abdomen sensible y con defensa muscular. Las pruebas de laboratorio del ingreso revelaron leucocitosis de $15850 \mathrm{x} \mathrm{mm}^{3}$, hemoglobina de $11,9 \mathrm{~g} /$ dL y PCR de 59,4 mg/L. Las otras pruebas de laboratorio no informaron hallazgos específicos. La TAC abdominal de urgencia, permitió verificar la existencia de una colección abdominal de aspecto masiforme, con realce de la pared abdominal anterior inferior izquierda y material lineal de alta densidad dentro de la masa inflamatoria. Se realizó una laparotomía exploradora y se encontró un absceso de aproximidamente 8 $\mathrm{cm}$ de diámetro mayor, localizado en la pared anterior del ciego, con adhesión a la pared abdominal. Después de drenar el absceso (350 cc de pus cremoso de mal olor), se encontró una perforación cecal y un CE firme y afilado de unos $5 \mathrm{~cm}$, compatible con un mondadientes. Se realizó una hemicolectomía derecha, ileotransverso anastomosis y aseo de la cavidad peritoneal con solución salina. La paciente tuvo un curso postoperatorio adecuado, sin somplicaciones y fue dada de alta al $5^{\circ}$ día con tránsito intestinal normal. Interrogada de forma dirigida, la paciente admitió haber participado de un cóctel, en el que había consumido "cositas de picar" y bebidas alcohólicas una semana antes de la consulta.

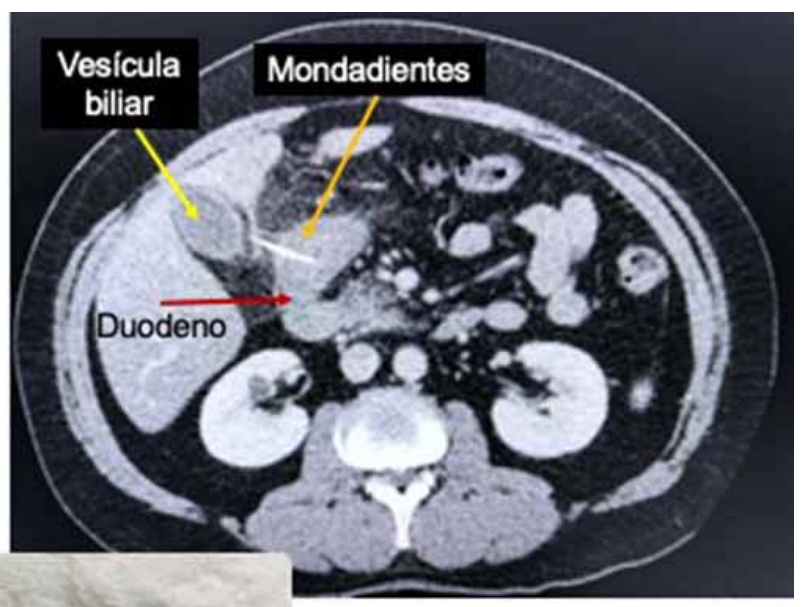

Fig. 1. Imágenes tomográficas de paciente con mondadientes que ha perforado la segunda porción del duodeno; y se encuentra en directa relación con la vesícula biliar. Una vez intervenida la paciente, el mondadientes es extirpado y se aprecia deteriorado en su estructura. 
Caso 3: Sujeto masculino de 72 años, hospitalizado en urgencias por un cuadro de tres días de evolución caracterizado por dolor abdominal, compromiso del estado general, deshidratación y fiebre. Antecedentes de hipertensión arterial sin tratamiento conocido. A la exploración del ingreso, se verificó un paciente con taquicardia $(102 \times \mathrm{min})$, febre $\left(\mathrm{t}^{\circ}\right.$ axilar de $\left.38,9^{\circ} \mathrm{C}\right)$, hipotensión $(95 / 60 \mathrm{mmHg})$, abdomen tenso y sensible. Las pruebas de laboratorio del ingreso revelaron leucocitosis de $16550 \mathrm{x} \mathrm{mm}^{3}$, hemoglobina de $12,2 \mathrm{~g} /$ dL y PCR de 47,2 mg/L. Las otras pruebas de laboratorio no informaron hallazgos específicos. La ecotomografía abdominal de urgencia, permitió verificar una colección abdominal con aspecto de absceso, de $12 \mathrm{~cm}$ de diámetro mayor, localizada en relación con el ángulo esplénico del colon, la curva mayor del estómago y el bazo. Se compensó al paciente, se inició terapia antibiótica de amplio espectro vía intravenosa. Cuatro horas después de su ingreso, se llevó a quirófano y tras recibir anestesia general, se realizó exploración abdominal, la que reveló la existencia de un gran absceso organizado, putrefacto, de aprox. $500 \mathrm{cc}$, localizado en el cuadrante superior izquierdo del abdomen, con rotura del bazo y perforación del colon izquierdo. Al disecar el absceso, apareció un $\mathrm{CE}$ afilado de consistencia plástica que resultó ser un mondadiente (Fig. 2). La cirugía consistió en esplenectomía, hemicolectomía izquierda, colostomía transversa y aseo peritoneal con solución salina. El paciente se recuperó de forma adecuada, fue dado de alta al $5^{\circ}$ día postoperatorio. Interrogado de forma dirigida, el paciente reconoció haber participado de una comida copiosa y abundante ingestión de alcohol 4 días antes de la consulta; en la que utilizó mondadientes para "picar carnes asadas". Ocho meses después se reconstituyó el tránsito colónico mediante colo-colo anastomosis, sin inconvenientes.

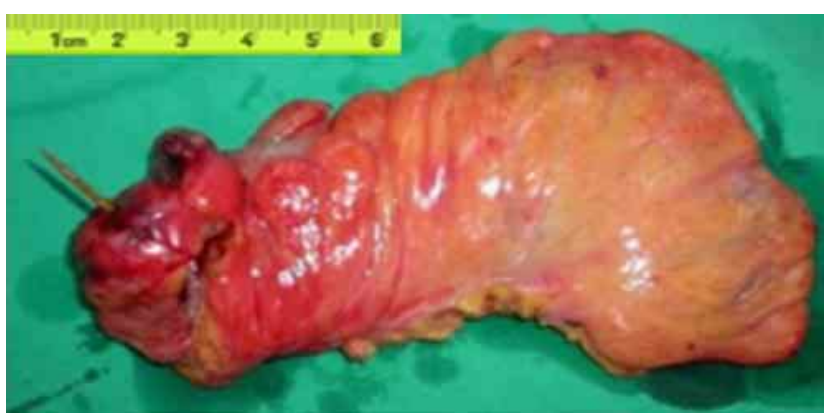

Fig. 2. Pieza de colectomía izquierda, en la que se aprecia el mondadientes perforando completamente la pared colónica.

\section{DISCUSIÓN}

La PI causada por IM suele ocurrir con mayor frecuencia en puntos de angulación fisiológica o zonas estenóticas del tracto digestivo ( $\mathrm{Su}$ et al.). Existe eviden- cia que señala que hasta el $83 \%$ de PI por CE ocurren en el ileon distal (Song et al., 2020). No obstante ello, existe evidencia de perforación duodenal (Ragazzi et al., 2010; Hashash et al.; Gardner et al., 2017), como ocurrió en uno de los casos reportados en este artículo.

Pero, se estima que la causa más común de PI por CE es por espinas; aunque su incidencia varía según los hábitos alimenticios de ingerir pescado (Goh et al., 2006; Wu et al., 2014).

En general, los pacientes con PI por CE ingerido consultan en servicios de urgencias por cuadros de abdomen agudo, peritonitis, abscesos, fístulas, obstrucción intestinal o hemorragia gastrointestinal (Syrakos et al., 2008; Dente et al., 2010; Gardner et al.).

Los pacientes habitualmente no informan la ingestión de un CE, lo que, junto con un perfil clínico a menudo confuso, puede complicar y retrasar el diagnóstico (Zouros et al., 2014). Los que son más susceptibles a la ingestión de $\mathrm{CE}$ son ancianos, alcohólicos, pacientes psiquiátricos, y usuarios de prótesis dentales. Estas últimas, reducen la sensibilidad táctil del paladar, lo que altera la capacidad de detectar objetos pequeños en la cavidad oral (Nicolodi et al.), y han sido un factor reportado en hasta el $80 \%$ de los casos de ingestión accidental de CE (Goh et al.).

Los CE ingeridos rara vez se detectan por radiología convencional, porque suelen ser de dimensiones pequeñas y baja radiopacidad (Yang et al., 2017; Lim et al.); así como porque a menudo están ocultos por gas intestinal. En un estudio de 358 pacientes que habían ingerido una espina de pescado, la radiología convencional tuvo una sensibilidad de 32\% (Ngan et al., 1990).

En la evaluación de pacientes con abdomen agudo, la TAC juega un rol fundamental, pues tiene sensibilidad de 95\% y $86 \%$ para la identificación y localización de la PI respectivamente; por lo que se considera una herramienta importante para los cirujanos para planificar la cirugía (Cadenas Rodríguez et al., 2013). También tiene alta sensibilidad para detectar CE pequeños y calcificados (huesos de pescado y pollo); CE no calcificados, como en estos casos, un mondadientes; e identificar áreas de estenosis inflamatorias o neoplásicas del tracto digestivo, que predisponen a la impactación de un CE (Lovece et al., 2014; Zouros et al.). Respecto de los mondadientes, se ha demostrado que su atenuación puede variar en función de la cantidad de aire y líquido dentro de la madera que los compone. Cuando se ingieren, suelen estar secos y llenos de aire, con un coeficiente de atenuación bajo; y debido a la absorción de fluidos, ésta aumenta después de unos días (Kuzmich et al., 2015). 
La estrategia de tratamiento depende de la ubicación del CE en el tracto digestivo y la presencia o ausencia de complicaciones como perforación, hemorragia y obstrucción. Los CE ubicados en el esófago o el estómago se extirpan preferentemente por vía endoscópica, mientras que los que se encuentran en el intestino delgado y colon, se tratan quirúrgicamente; ya sea por laparotomía o laparoscopia, con resección segmentaria del asa afectada (Syrakos et al.; Sarmast et al., 2012; Lim et al.).

En una búsqueda sistemática referente a PI por IM, se encontraron 131 artículos que aportaron 157 casos, cuyas características principales se resumen en la Tabla I y la Figura 3 (Ragazzi et al.; Sarmast et al.; Lovece et al.; Nic 'iforovic et al., 2014; Steinbach et al.; Zeng et al., 2014; Zouros et al.; Zelones et al., 2015; da Mota et al., 2016; Birindelli et al., 2016; Çapkinoglu et al., 2017; Kumar et al., 2017; Sarici et al., 2017; Turaihi et al., 2017; Yang et al.; Lai et al., 2018; Lin et al., 2018; Depoorter et al., 2019; Lim et al.; Ossola \& Galafassi, 2019).

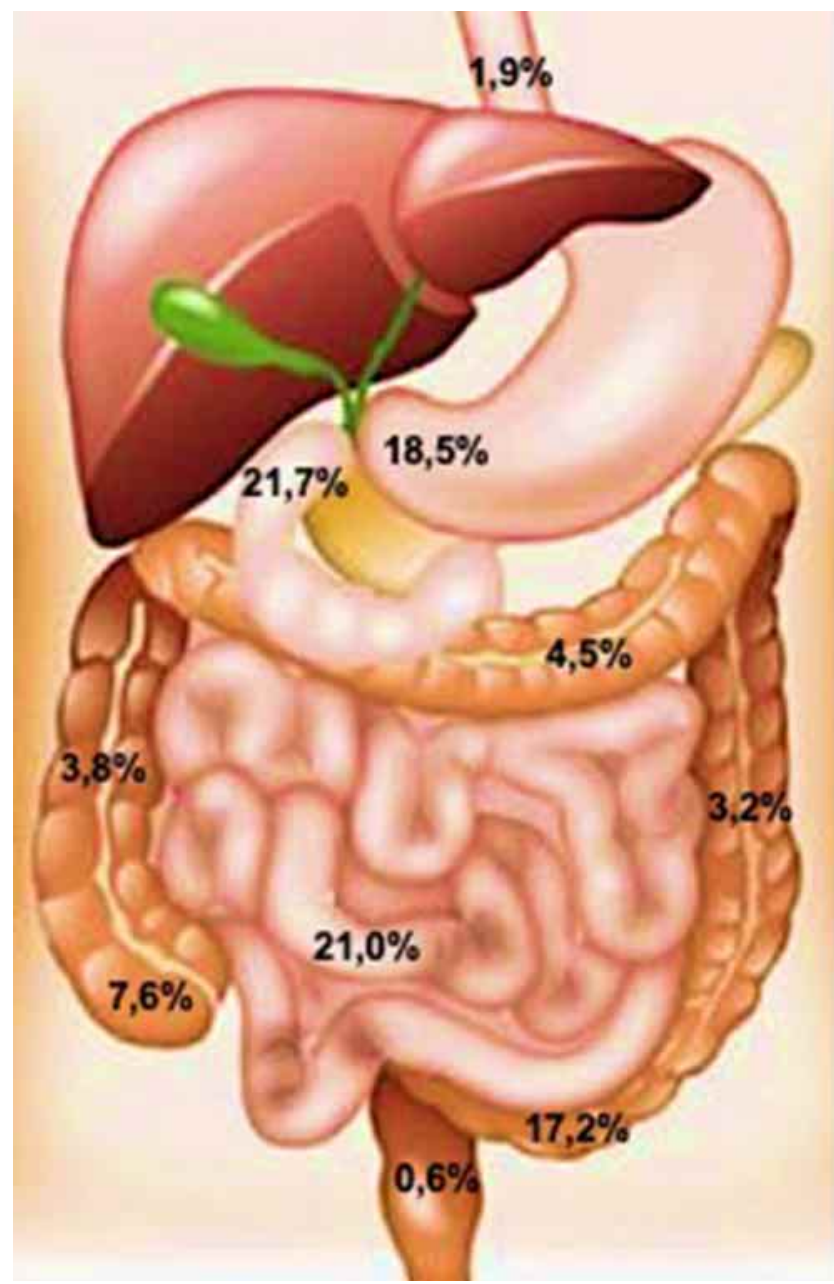

Fig. 3. Distribución porcentual de la localización de las perforaciones por mondadientes en 157 pacientes registrados (Tabla I).
Tabla I. Experiencia Reportada con PI por IM $(\mathrm{N}=157)$

\begin{tabular}{lc}
\hline \multicolumn{1}{c}{ Variables } & $\begin{array}{c}\text { Promedio } \\
\text { (mínimo-máximo) }\end{array}$ \\
\hline Edad (años) & $51,7(5-92)$ \\
Sexo masculino (\%) & 71,2 \\
Tiempo síntomas (días) & $6,7(2$ a 60) \\
Acceso terapéutico (\%) & \\
$\quad$ Laparotomía & 57,1 \\
$\quad$ Laparoscopía & 16,0 \\
$\quad$ Endoscopía & 26,9 \\
Tiempo quirúrgico (minutos) & $62(45-90)$ \\
Hospitalización (días) & $7,2(4-25)$ \\
Mortalidad (\%) & 8,3 \\
\hline
\end{tabular}

MANTEROLA, C. Results of surgical treatment of intestinal perforation caused by toothpick ingestion. Contribution of 3 cases. Int. J. Morphol., 38(5):1212-1216, 2020.

SUMMARY: Toothpick ingestion (TPI) is a rare event, but can cause intestinal perforation (IP), peritonitis, sepsis, and even death (approximately 157 cases have been published worldwide). This fact determines the need for surgical intervention in most of these patients. The aim of this manuscript was to report the results of the surgical treatment in 3 cases of IP by TPI. The report involves the following: Case 1: A 52-year-old man, with duodenal and gallbladder perforation. Case 2: A 54-year-old woman with cecal perforation. Case 3: A 72-year-old man, with perforation of the left colon and splenic rupture. All were hospitalized for abdominal pain and fever. In two of the cases the diagnosis was verified by tomography and in one by ultrasound. All three patients underwent laparotomy. In the first case (1), cholecystectomy and duodenal suture were performed; in the second case (2) right hemicolectomy and ileo-transverse anastomosis were performed, and in the third case (3), Left hemicolectomy and splenectomy were performed. Toothpick was found in all cases. The patients in this report all evolved satisfactorily without postoperative complications. In conclusion, TPI is a surgical emergency. IP are common and the associated peritonitis is high. Prognosis depends on early diagnosis and timely treatment.

KEY WORDS: Intestinal Perforation[Mesh]; Intestinal Perforation/etiology[Mesh]; Toothpicks; Intestinal Perforation/surgery[Mesh]

\section{REFERENCIAS BIBLIOGRÁFICAS}

Birindelli, A.; Tugnoli, G.; Biscardi, A. \& Di Saverio, S. Retroperitoneal colonic perforation from a foreign body--'Cannibalization' effect of a toothpick--Video vignette. Colorectal Dis., 18(3):319-21, 2016.

Cadenas Rodríguez, L.; Martí de Gracia, M.; Saturio Galán, N.; Pérez Dueñas, V.; Salvatierra Arrieta, L. \& Garzón Moll, G. Use of multidetector computed tomography for locating the site of gastrointestinal tract perforations. Cir. Esp., 91(5):316-23, 2013.

Çapkınoglu, E.; Özsay, O.; Örsel, A. \& Karaisli, S. Toothpick ingestion causing rectum perforation. Turk. J. Gastroenterol., 28(6):540-1, 2017. 
da Mota, F. F.; Lisboa, T. V.; da Costa, B. X. M.; de Queiroz, F. L.; Mansur, E. S.; de Almeida Paiva, R. \& França Neto, P. R. A novel clip-assisted method for endoscopic removal of an impacted toothpick from the colon. Endoscopy, 48 Suppl. 1:E259-60, 2016.

Dente, M.; Cilurso, F.; Fornari, L.; Morano, C.; Moretti, M.; Neri, T.; Solinas, L.; Tiberi, R.; Anastasio, G.; Santi, F.; et al. Intestinal perforation due to accidental ingestion of foreign bodies. G. Chir, 31(1-2):38-41, 2010.

Depoorter, L.; Billiet, T.; Verhamme, M. \& Van Moerkercke, W. A toothpick a day, keeps the doctor away? Acta Gastroenterol. Belg., 82(1):97-8, 2019.

Gardner, A. W.; Radwan, R. W.; Allison, M. C. \& Codd, R. J. Double duodenal perforation following foreign body ingestion. BMJ Case Rep., 2017:bcr2017223182, 2017.

Goh, B. K. P.; Tan, Y. M.; Lin, S. E.; Chow, P. K. H.; Cheah, F. K.; Ooi, L. L. P. J. \& Wong, W. K. CT in the preoperative diagnosis of fish bone perforation of the gastrointestinal tract. AJR Am. J. Roentgenol., 187(3):710-4, 2006.

Hashash, J. G.; Kaufman, L. C.; Chedid, V. \& Fasanella, K. Duodenal perforation from a pen. Endoscopy, 47 Suppl. 1 UCTN:E121-2, 2015.

Kumar, S.; Mehdipour, R.; Arachchi, A. \& Cass, A. Toothpick impaction in a sigmoid diverticulum. BMJ Case Rep., 2017:bcr2017219198, 2017.

Kuzmich, S.; Burke, C. J.; Harvey, C. J.; Kuzmich, T.; Andrews, J.; Reading, N.; Pathak, S. \& Patel, N. Perforation of gastrointestinal tract by poorly conspicuous ingested foreign bodies: radiological diagnosis. $\mathrm{Br}$. J. Radiol., 88(1050):20150086, 2015.

Lai, C. M. S. \& Lui, T. H. Small bowel perforation by toothpick. BMJ Case Rep., 2018:bcr2018225258, 2018.

Lim, D. R.; Kuk, J. C.; Kim, T. \& Shin, E. J. Surgery for intra-abdominal abscess due to intestinal perforation caused by toothpick ingestion: two case reports. Medicine (Baltimore), 98(36):e17032, 2019.

Lin, N.; Lin, L.; Wu, W.; Yang, W.; Cai, Z.; Hong, J. \& Wang, Y. Successful diagnosis and treatment of ingested wooden toothpicks. Two case reports. Medicine (Baltimore), 97(5):e9710, 2018.

Lovece, A.; Asti, E.; Sironi, A. \& Bonavina, L. Toothpick ingestion complicated by cecal perforation: case report and literature review. World J. Emerg. Surg., 9(1):63, 2014.

Lunsford, K. E. \& Sudan, R. Small bowel perforation by a clinically unsuspected fish bone: laparoscopic treatment and review of literature. J. Gastrointest. Surg., 16(1):218-22, 2012.

Ngan, J. H.; Fok, P. J.; Lai, E. C.; Branicki, F. J. \& Wong, J. A prospective study on fish bone ingestion. Experience of 358 patients. Ann. Surg., 211(4):459-62, 1990.

Nic'iforovic, D.; Spasic, A.; Turkalj, I. \& Vanhoenacker, F. M. Toothpick perforation of the caecum. JBR-BTR., 97(1):47, 2014.

Nicolodi, G. C.; Trippia, C. R.; Caboclo, M. F. F. S.; de Castro, F. G.; Miller, W. P.; de Lima, R. R.; Tazima, L. \& Geraldo, J. Intestinal perforation by an ingested foreign body. Radiol. Bras., 49(5):295-9, 2016.

Ossola, L. \& Galafassi, J. Dual site intestinal perforation due to toothpick. G. Chir., 40(4):330-3, 2019.

Ragazzi, M.; Delcò, F.; Rodoni-Cassis, P.; Brenna, M.; Lavanchy, L. \& Bianchetti, M. G. Toothpick ingestion causing duodenal perforation. Pediatr. Emerg. Care, 26(7):506-7, 2010.

Sarici, I. S.; Topuz, O.; Sevim, Y.; Sarigoz, T.; Ertan, T.; Karabıyı, O. \& Koc, A. Endoscopic management of colonic perforation due to ingestion of a wooden toothpick. Am. J. Case Rep., 18:72-5, 2017.

Sarmast, A. H.; Showkat, H. I.; Patloo, A. M.; Parray, F. Q.; Lone, R. \& Wani, K. A. Gastrointestinal tract perforations due to ingested foreign bodies; a review of 21 cases. BJMP., 5(3):a529, 2012.

Song, J.; Yang, W.; Zhu, Y.; Fang, Y.; Qiu, J.; Qiu, J.; Lin, L.; Wu, W.; Lin, C. \& Wang, Y. Ingested a fish bone-induced ileal perforation: a case report. Medicine (Baltimore), 99(15):e19508, 2020.

Steinbach, C.; Stockmann, M.; Jara, M.; Bednarsch, J. \& Lock, J. F. Accidentally ingested toothpicks causing severe gastrointestinal injury: a practical guideline for diagnosis and therapy based on 136 case reports. World J. Surg., 38(2):371-7, 2014.
Su, F. J.; Lin, I. L.; Yan, Y. H. \& Huang, C. L. Fishbone perforation of the gastrointestinal tract in patients with acute abdominal pain: diagnosis using plain film radiography. BMJ Case Rep., 2014:bcr2014204201, 2014.

Syrakos, T.; Zacharakis, E.; Antonitsis, P.; Zacharakis, E.; Spanos, C.; Georgantis G. \& Kiskinis, D. Surgical intervention for gastrointestinal foreign bodies in adults: a case series. Med. Princ. Pract., 17(4):276-9, 2008.

Turaihi, H.; Assam, J.; Zanfes, J. \& Thambi-Pillai, T. Laparoscopic treatment of a pyogenic hepatic abscess caused by transmural duodenal perforation of a toothpick. S. D. Med., 70(8):369-71, 2017.

Wu, C. X.; Wu, B. Q.; Duan, Y. F.; Sun, D. L. \& Jiang, Y. Rare case of omentum-wrapped abscess caused by a fish bone penetrating the terminal ileum. World J. Gastroenterol., 20(32):11456-9, 2014.

Yang, Z.; Wu, D.; Xiong, D. \& Li, Y. Gastrointestinal perforation secondary to accidental ingestion of toothpicks: A series case report. Medicine (Baltimore), 96(50):e9066, 2017.

Zelones, J.; Kiyama, L.; Sandler, B. J. \& Kane, C. J. Sigmoid perforation due to toothpick ingestion leading to hydronephrosis and urosepsis. Am. Surg., 81(5):E232-3, 2015.

Zeng, H. Z.; Wang, Q. M.; Liu, W.; Mou, Y.; Yi, H.; Wang, S. F. \& Hu, B. Kidney injury and hematuria as a result of duodenal perforation by an ingested toothpick. Endoscopy, 46 Suppl. 1 UCTN:E559-60, 2014.

Zouros, E.; Oikonomou, D.; Theoharis, G.; Bantias, C. \& Papadimitropoulos, K. Perforation of the cecum by a toothpick: report of a case and review of the literature. J. Emerg. Med., 47(6):e133-7, 2014.

\section{Direccion para correspondencia:}

Dr. Carlos Manterola

Centro de Exelencia en Estudios Morfológicos y Quirúrgicos Universidad de La Frontera

Temuco

CHILE

\section{Email: carlos.manterola@ufrontera.cl}

Recibido : 18-02-2020

Aceptado: 22-04-2020 